\title{
A Review on Photo Voltaic MPPT Algorithms
}

\author{
Saravana Selvan*, Pratap Nair*, Umayal** \\ * Faculty of Engineering and Computer Technology, AIMST University, Bedong, Kedah, Malaysia \\ ** Graduated in Anna University Chennai, India
}

\begin{tabular}{l}
\hline \hline Article Info \\
\hline Article history: \\
Received Aug 12, 2015 \\
Revised Nov 25, 2015 \\
Accepted Dec 16, 2015 \\
\hline
\end{tabular}

Keyword:

Direct

IndirectIntelligence techniques MPPT algorithms

PV array

\begin{abstract}
A photovoltaic generator exhibits nonlinear voltage-current characteristics and its maximum power point varies with solar radiation and cell temperature. A Dc/Dc power converter is used to match the photovoltaic system to the load and to operate the PV (photo voltaic) cell array at maximum power point. Maximum Power Point Tracking (MPPT) is a process which tracks one maximum power point from PV array input, varying the ratio between the voltage and current delivered to get the most power it can. There are different techniques proposed with lot of algorithms are being used in the MPPT controller to extract the maximum power. It is very difficult for the photo voltaic designers, researchers and academic experts to select a particular MPPT technique for a particular application which requires the background knowledge and comparative features of various MPPT algorithms. This paper will be avaluable source for those who work in the photo voltaic generation, so its objective is to review the main MPPT algorithms in practice and analyzes the merits and demerits with various factors.
\end{abstract}

Copyright () 2016 Institute of Advanced Engineering and Science. All rights reserved.

\section{Corresponding Author:}

Saravan Selvan,

Faculty of Engineering and Computer Technology,

AIMST University,

Bedong, Kedah, Malaysia

Email: saravanan@aimst.edu.my

\section{INTRODUCTION}

Our world is witnessing a lot of energy crisis and environmental pollution, to solve it the renewable energy is the alternate sources of energy. Solar energy has the advantages of maximum reserve, in exhaustibleness, and is free from geographical restrictions, thus making photovoltaic (PV) technology a popular research topic. Currently more research works has been focussed on how to extract more power effectively from the PV cells. At constant uniform irradiance, the PV array has a current- voltage (I-V) characteristic like that shown in figure 1. In that curve, there is a unique point called the maximum power point (MPP), at which the array operates at maximum efficiency and produces output power. But the problemis when the PV array is directly connected to a load, the system operating point is not exactly at the MPP rather than that the intersection of the I-V curve of the PV array and load line can happen as shown in figure 1. And also at the time of partially shaded condition or change in weather conditions i.e, solar irradiation and atmospheric temperature, the system can not operate at the PV array MPP. It is noted that under partial shading conditions, the P-V characteristics of the PV array get more complex and bear multiple peaks as shown in figure 1.

To overcome this problem, Maximum power point techniques (MPPT) are employed in photovoltaic systems to make full utilization of PV array output power [1-5]. Maximum Power Point Tracking (MPPT) is a process which tracks one maximum power point (MPP) from photo voltaic (PV) array input. The main challenge by MPPT techniques is to automatically find the voltage $\mathrm{V}_{\mathrm{MPP}}$ or current $\mathrm{I}_{\mathrm{MPP}}$ at which a PV array should operate to obtain the maximum power output $\mathrm{P}_{\mathrm{MPP}}$ under a given temperature and irradiance [1-2]. 
In order to locate and track the MPP of the PV array, MPPT algorithms are used in the technique. Several techniques and algorithms have been proposed in this area to overcome the drawbacks of the solar PV system [3-5]. The Maximum Power Point Tracking (MPPT) is usually used as online control strategy to track the maximum output power operating point of the Photovoltaic generation (PVG) for different operating condition of insolation and temperature of the PVG. Hohm D, Ropp M compares and evaluates the percentage of power extraction with MPPT and without MPPT [22]. Her-TerngYau [23] uses the MPPT techniques with the PV system for optimal charging of Li-ion Battery.The authors [24] [25] proposes the modified two basic MPPT algorithms like perturb and observe and Incremental conductance which shows the later one is very complex to implement and the former one is very easy to implement but not reliable. Salam Zainal [8] analyzes the performance of soft computing based MPPT algorithms under partial shaded condition which creates a transient output in his work. The application of intelligent techniques like fuzzy logic, neural network in the MPPT technique to track the MPP is achieved by the researhers in this paper [27].

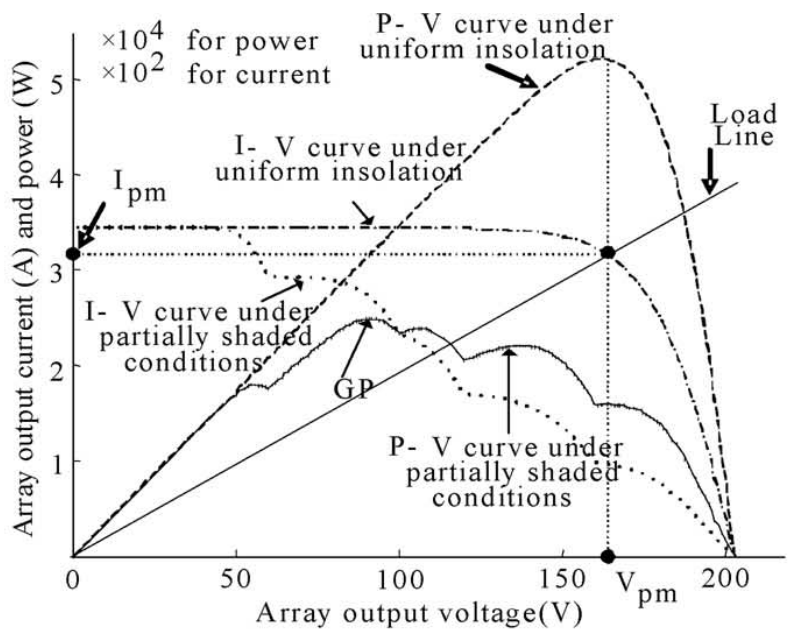

Figure 1. PV-IV Characteristics of uniform solar irradiance and under partial shaded condition [1]

There are lot of MPPT tracking techniques are in practice and the comparison of most of the MPPT techniques with respect to the amount of energy extracted from the PV panel have been proposed in the literature [1-5].With this in mind, there are many MPPT algorithms have been developed and intensive research has been carried out to optimize the techniques. The paper reviews the various MPPT algorithm techniques that have been used for Photovoltaic system at changes in irradiance and temperature. It discusses the technical aspects, merits, demerits and analyzes the performance of each method. It is very difficult for the Photo voltaic designers, researchers and academic experts to select a particular MPPT technique for a particular application which requires the background knowledge and comparative features of various MPPT algorithms. Obviuously this paper would be a valuable source for those who require more information to design an improved MPPT for inverters as well as it can also provide a convenient reference for future work in PV power generation.

In this paper, the section 2 discusses the basic equivalent circuit and nonlinear characteristics of PV panel is discussed. Section III describes the classification of MPPT Techniques. Section III, IV \& V reviews the working principle of various MPPT algorithms based on the technique and being compare the performance of each techniques with critical parameters.

\section{RESEARCH METHOD}

A solar PV cell is basically a p-n junction fabricated in a thin wafer of semiconductor. The electromagnetic radiation of solar energy can be directly converted to electricity through photovoltaic effect. Being exposed to the sunlight, photons with energy greater then the band-gap energy of the semiconductor creates some electron-hole pairs proportional to the incident irradiation. To achieve higher voltage and current, multiple cells are used as needed. The PV cell can be represented by a simple equivalent circuit with the series resistance Rs represents the internal losses due to the current flow, whereas the shunt resistance 
Rsh corresponds to the leakage current to the ground and it is normally ignored. The output current is a function of solar radiation, temperature, wind speed and coefficients that are particular to the cell technology. The authors [6-7] presented a detailed analysis of solar cell and its electrical equivalent model is created in Matlab/Simulink. The net current equation (Eqn 1.1) of the PV cell is the difference between the photocurrent Iph and the normal diode current ID.

$$
I=I_{p h}-I_{D}=I_{p h}-I_{o}\left(\exp \frac{e\left(V+I R_{s}\right)}{m k T_{c}}-1\right)
$$

where:

$\mathrm{m}$ - Idealizing factor

$\mathrm{k}$ - Boltzmann's gas constant

Tc- Absolute temperature of the cell

e- Electronic charge

V- Voltage impose across the cell

Io -Dark saturation current

PV modules have unique current v/s voltage (I-V) characteristics. From the P-V and I-V characteristics, as shown in figure $2 \mathrm{a} \& \mathrm{~b}$ [5], it is clear that the PV systems must be operated at a maximum power point (MPP) of specific current and voltage values so as to increase the PV efficiency. The voltage that corresponds to the module maximum power varies with temperature and insolation variations, so a MPP tracking system is needed to ensure that we stay as close as possible to the maximum power point.

For any PV system, the output power can be increased by tracking the MPP (Maximum Power Point) of the PV module by using a controller connected to a dc- dc converter (usually boost converter). However, the MPP changes with insolation level and temperature due to the nonlinear characteristic of PV modules. Each type of PV module has its own specific characteristic. In general, there is a single point on the V-I or V-P curve, called the Maximum Power Point (MPP), at which the entire PV system operates with maximum efficiency and produces its maximum output power. This point can be located with the help of MPPT (Maximum Power Point Trackers). PV system with MPPT controller has been shown in figure 3.

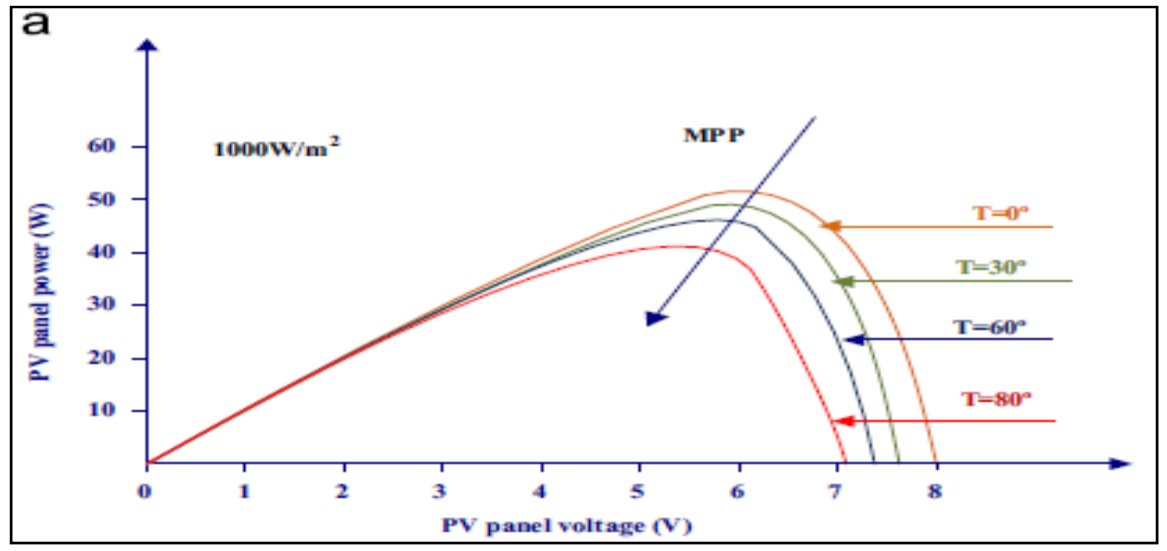

Figure 2. (a) PV characteristic at constant insolation and different temperatures [5] 


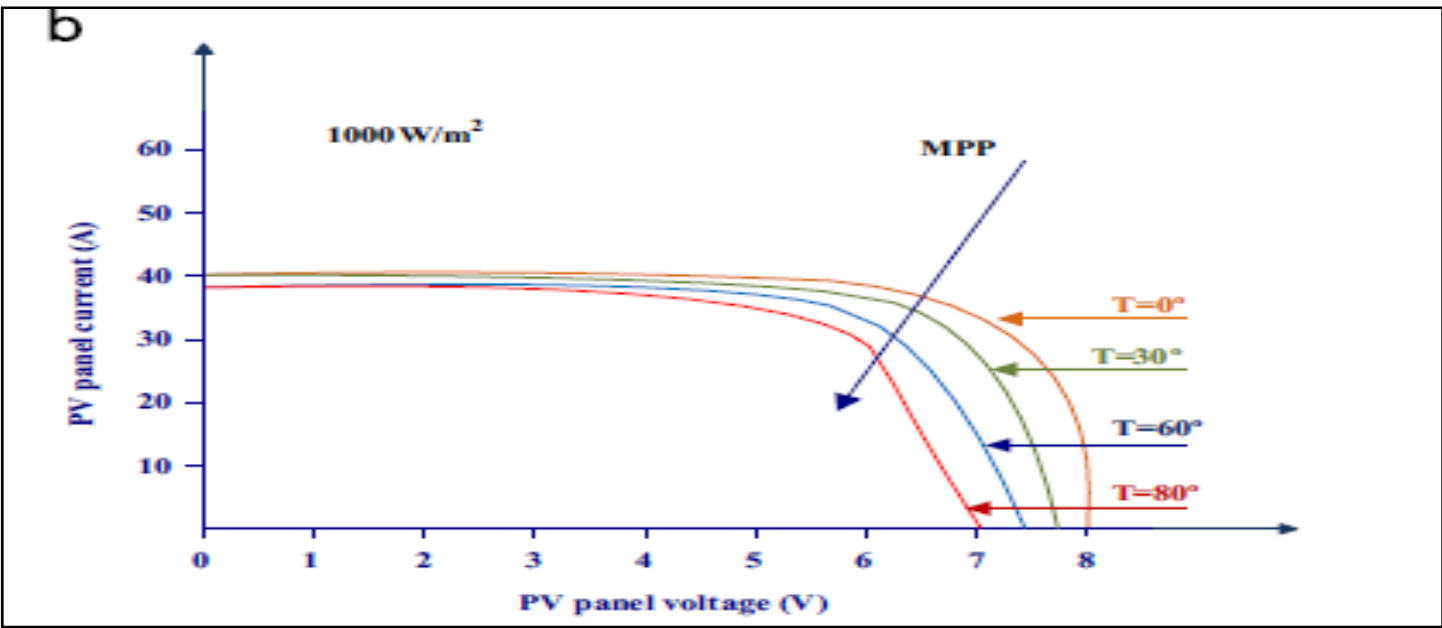

Figure 2. (b) IV characteristic at constant insolation and different temperatures [5]

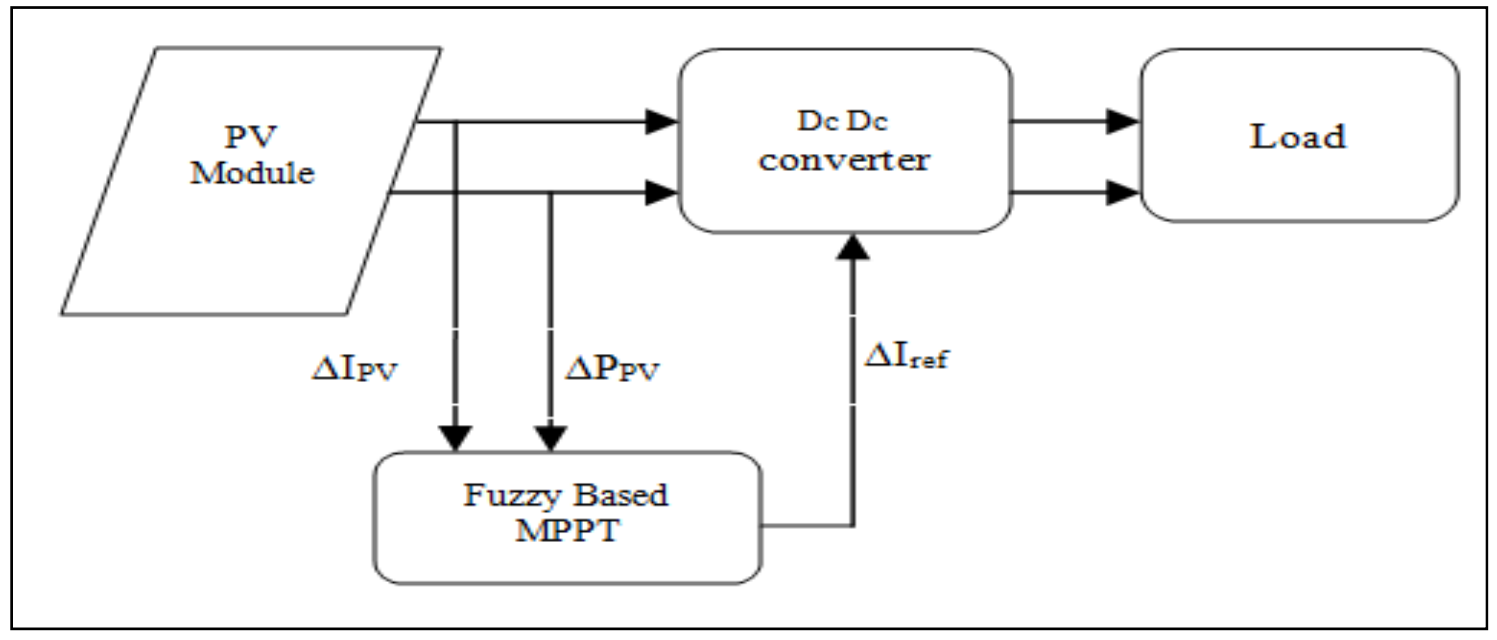

Figure 3. Block diagram of MPPT techniques [10]

\section{CLASSIFICATION OF MPPT TECHNIQUES}

The classification of commonly used MPPT technique in the PV system is shown in figure 4 . The following section will briefly reviewed the main MPPT techiniques. 


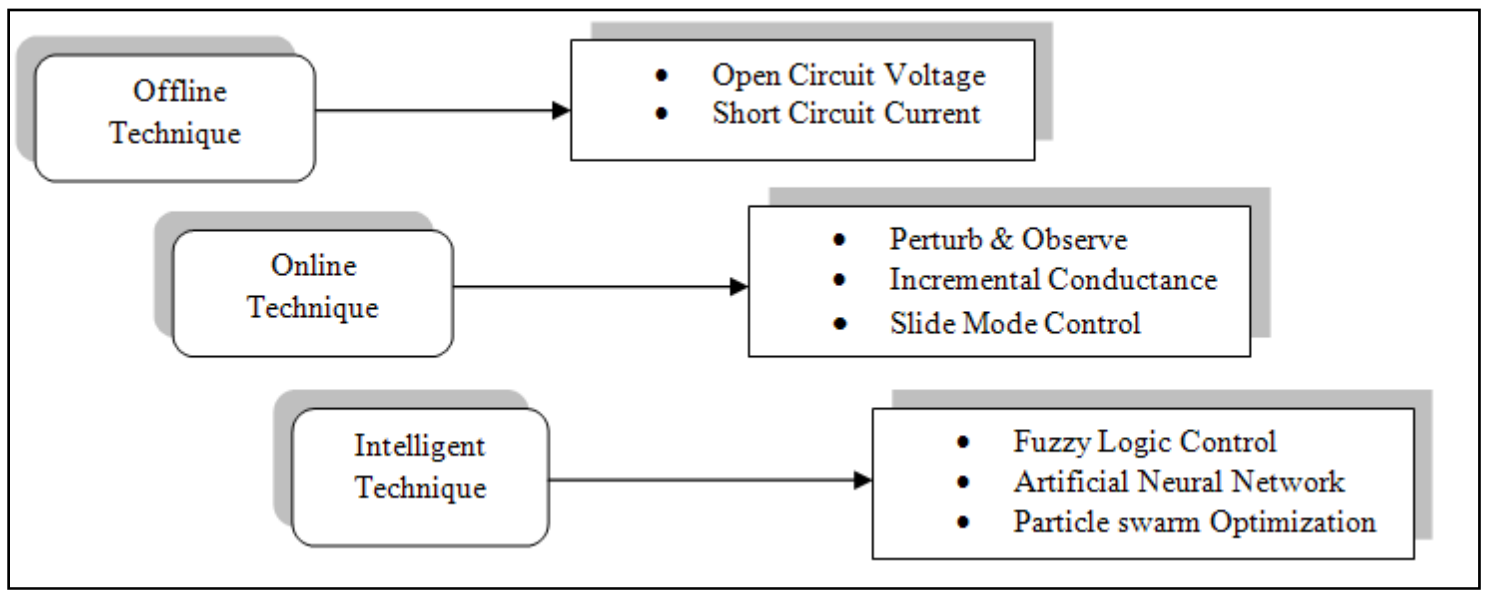

Figure 4. Classification of commonly used MPPT Technique

\subsection{Offline Technique}

The offline technique is commonly called as indirect technique since this algorithm is worked in offline i.e. not on the real time. In this technique, there is a necessity of prior requirement of particular PV panel data such as the mathematical modeling, I-V, P-V curve, etc. The basic indirect techniques of MPPT are open circuit voltage and Short circuit current method [5]. In both the methods, a DC/DC converter with the driver circuit is controlled by the microcontroller or DSP operates either in Buck/Boost mode is required. The duty cycle of the switching converter can be varied based on the computation of the open circuit voltage or short circuit current to track the maximum power point (MPP).

\subsubsection{Open Circuit Voltage Technique}

In this technique, the value of Vmpp under different solar insolation and temperature is calculated approximately by the equation:

$$
\mathrm{Vmpp}=\mathrm{K} 1 \mathrm{Voc}
$$

Where Voc is the open circuit voltage, $\mathrm{K} 1$ is the coefficient value ranges from 0.71 to 0.9 depending upon the characteristics of the panel. The value of Vmpp is computed by measuring the value of Voc periodically.

\subsubsection{Short Circuit Current Technique}

This technique is also similar to the previous indirect method instead of voltage controlled here the computation is based on the value of short circuit current Isc of PV panel. The peak value of current Impp is computed by the following equation as:

$$
\operatorname{Impp}=\mathrm{K} 2 \mathrm{Isc}
$$

Where $\mathrm{K} 2$ is the coefficient value ranges from 0.73 to 0.95 depending on the cell material and characteristics of the PV panel. By computing the values of Impp, at different sampled values of Isc, the duty cycle of the DC/DC converter is increased or decreased so that the output current becomes equal to Impp.The implementation of this algorithm results reviewed in the literature reports that regarding the cost wise is very effective but the performance wise is not good during different irradiance of sun and different operating temperature of the panel.

\subsection{Online Technique}

The popular online techniques used directly for the maximum power point are Perturb and Observe Technique (P\&O) and Incremental conductance Technique (INC) [1-4]. In the direct techniques, there will be an online measurement of voltage and measurement of the panel periodically for computing the duty cycle of the converter to track the maximum power point MPPT. 


\subsubsection{P\&O MPPT Algorithm}

This algorithm is an iterative technique and very simple one. It measures the panel operating voltage and current periodically and calculates the instantaneous power $\mathrm{p}(\mathrm{n})$. This instantaneous power compares with the previous output power $\mathrm{P}(\mathrm{n})-\mathrm{P}(\mathrm{n}-1)$, then the operating voltage $\mathrm{X}(\mathrm{n}+1)$ is determined and changed by changing the duty ratio of the converter $X(n+c)$ or $X(n-c)$. The sign of change of output power is also observed to track the MPP. If the output power increases by increasing the operating voltage, then the operating voltage is further perturbed in the same direction until it reaches the point of MPP or $d p / d v=0$. If the output power decreases by increasing the operating voltage, i.e the operating point is in the negative slope region of the PV curve. Then the operating voltage is perturbed in the reverse direction to track the MPP. The flow chart of the $\mathrm{P} \& \mathrm{O}$ algorithm is shown in the figure 5.

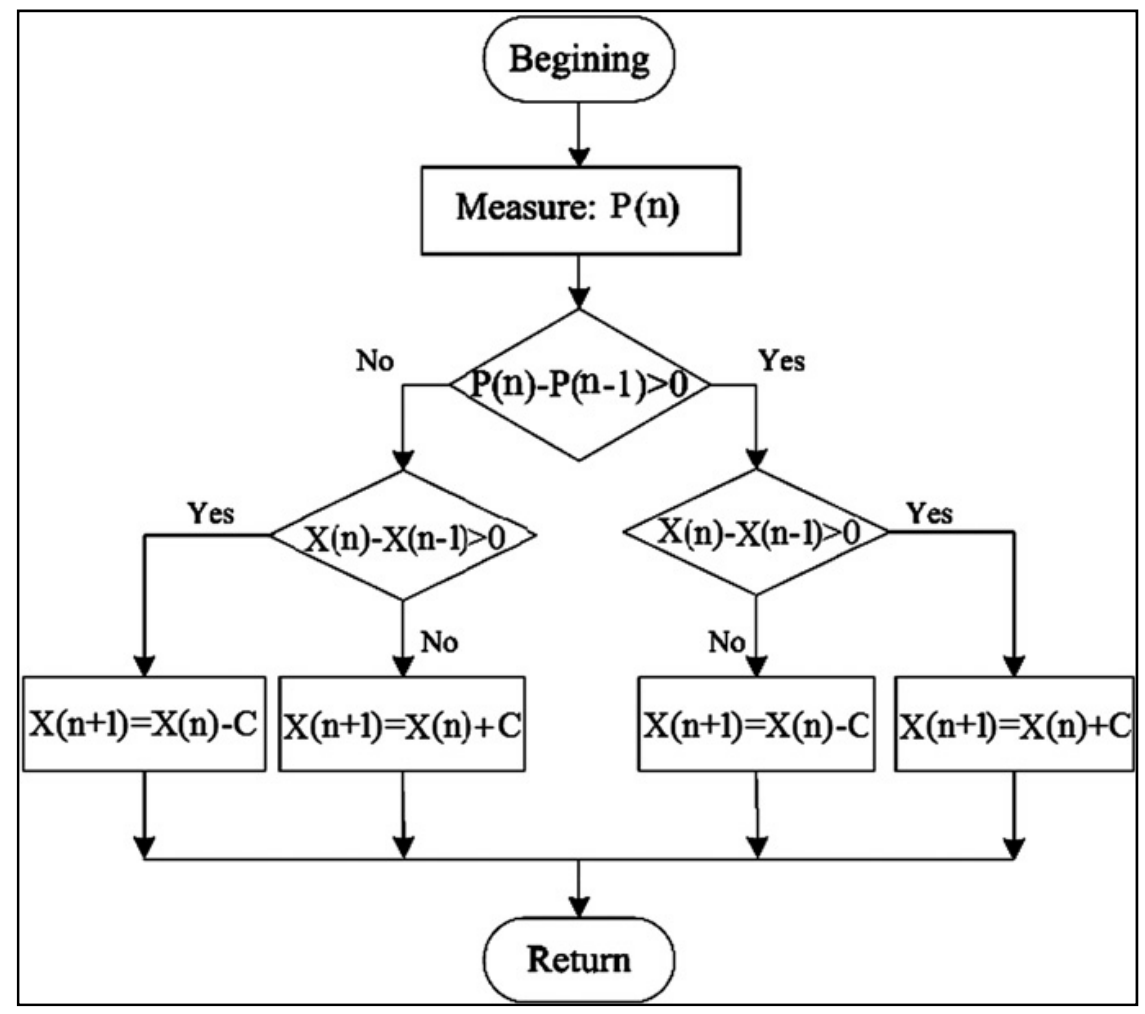

Figure 5. Flow chart of $\mathrm{P} \& \mathrm{O}$ algorithm [12]

The main advantage of this technique is very simple and easily implemented. But the disadvantage is the operating point is highly oscillated around the MPP point and not fixed on the exact MPP. The literature review reveals that if we increase the step size of the algorithm, the oscillations around the MPP will be more and dynamic. If we reduce the step size, the convergence speed will be very less and cannot able to track the MPP at various atmospheric conditions. Several researchers in the review suggested or modified the $\mathrm{P} \& \mathrm{O}$ algorithm to have better efficiency compare to the conventional.

\subsubsection{Incremental Conductance Algorithm (INC)}

This is a most popular MPPT algorithm and widely used. The disadvantages of the P\&O MPPT algorithm is overcome and eliminated by this INC technique but it is more complex and very difficult to implement. The theory of the incremental conductance is to determine the variation direction of the output terminal voltage of the PV modules by measuring and comparing the incremental conductance and instantaneous conductance of PV modules. If the value of incremental conductance and the instantaneous conductance are equal, then it represents the operating point reaches the MPP maximum power point. Compare to the offline techniques, the online techniques like incremental conductance (INC) algorithms are advantageous under rapidly changing atmospheric conditions but it involves complex calculation which increases the hardware requirement and cost. 
The basic concept of Incremental conductance on a PV curve is shown in figure 6. The slope of the $\mathrm{P}-\mathrm{V}$ curve is zero at the MPP, increasing on the left of the MPP and decreasing on the Right side of the MPP. The basic equations of this method are as follows.

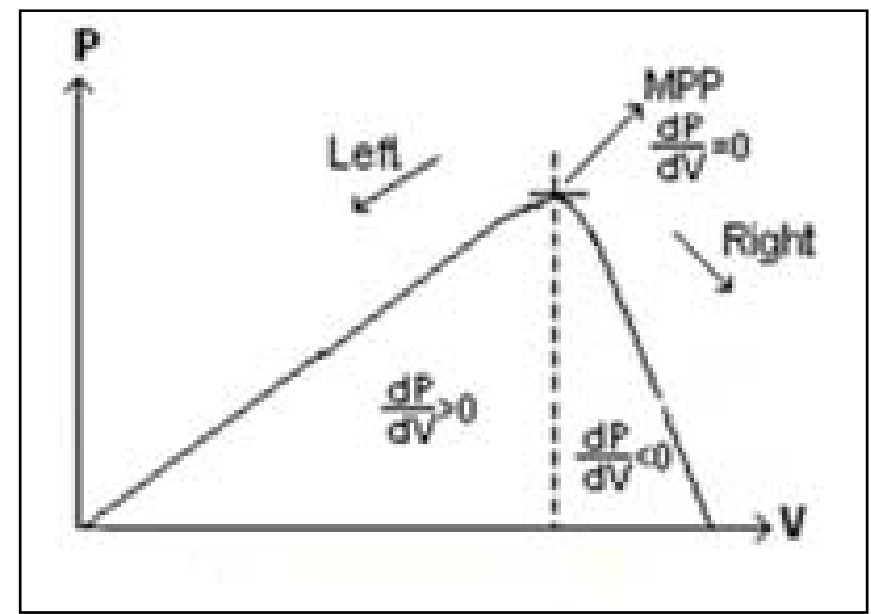

Figure 6. Concept of Incremental conductance algorithm

$$
\begin{array}{ll}
d P / d V=0 & \text { at } M P P \\
d P / d V>0 & \text { left of } M P P \\
d P / d V<0 & \text { right of } M P P \\
d P / d V=d(V I) / d(V)=I+V^{*} d I / d V
\end{array}
$$

Eqn. 3.3

The $\mathrm{dP} / \mathrm{dV}$ is defined as Maximum power point identifier factor. By utilizing this factor, the INC method is proposed to effectively track the MPP of PV module. The following definitions are considered to track the MPP.

$$
\begin{array}{ll}
\Delta \mathrm{I} / \Delta \mathrm{V}=-\mathrm{I} / \mathrm{V} & \text { at MPP, } \Delta \mathrm{Vn}=0 \\
\Delta \mathrm{I} / \Delta \mathrm{V}>-\mathrm{I} / \mathrm{V} & \text { left of MPP, } \Delta \mathrm{Vn}=+\delta \\
\Delta \mathrm{I} / \Delta \mathrm{V}<-\mathrm{I} / \mathrm{V} & \text { right of MPP }, \Delta \mathrm{Vn}=-\delta
\end{array}
$$

Eqn. 3.4

Eqn. 3.5

Eqn. 3.6

Where $\Delta \mathrm{Vn}$ is the change in the operating voltage of nth iteration and $\delta$ sign indicates the increase or decrease the step size of the converter. Consider the nth iteration of the algorithm as a reference, and then $\mathrm{n}+1$ iteration process can be determined by using the above equations. The MPPT regulates the PWM control signal of the DC/DC power converter until the condition: $(\mathrm{dI} / \mathrm{dV})+(\mathrm{I} / \mathrm{V})=0$ is satisfied. The Flow chart of incremental conductance MPPT algorithm is shown in figure 7. This technique gives good results during rapidly changing environment conditions with minimal oscillations. But the complex computation procedure of this algorithm makes the implementation very difficult and convergence towards the MPP is not accurate. 


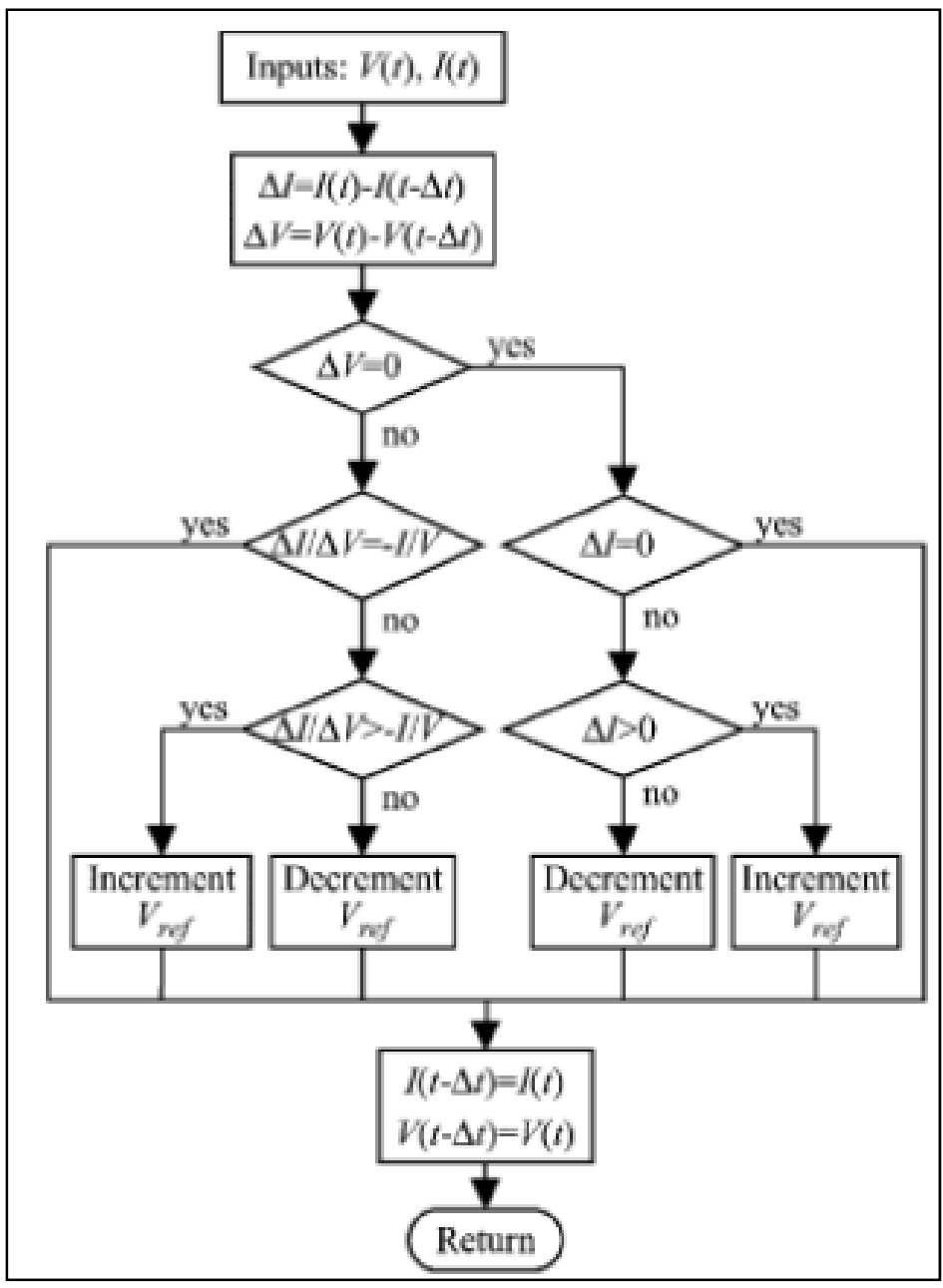

Figure 7. Flowchart of Incremental conductance algorithm [4]

\subsubsection{Slide Mode Control}

This control technique is used for non linear systems. It provides control of power converter which tracks the MPP under the condition of varying irradiance. Slide mode control algorithms is based on the on/off controlling the power switch of the Dc-Dc converter which continuously track the MPP, so the efficiency of this algorithm is very high. The MPPT speed will be faster when increasing the switching of the converter but the power output and voltage output fluctuation increases. The sliding mode control consists of sliding surface and control law design. The sliding surface is defined based on Incremental Conductance (IC) MPPT algorithm [9]. From the Incremental conductance algorithm.

$$
(\mathrm{dI} / \mathrm{dV})+(\mathrm{I} / \mathrm{V})=0
$$

The state equation s for the Buck-Boost converter is given by

$$
\frac{d I_{L}}{d t}=\frac{D \cdot V_{p}}{L}-\frac{(1-D) V_{o}}{L}
$$

Where, IL is the inductor current, $\mathrm{Vp}$ is the $\mathrm{PV}$ panel voltage,

$\mathrm{D}$ is the duty ratio of the switch, Vo is the ouput voltage.

The inversion of the equation (3.7) is given by

$$
-d V p / d I p=V p / I p
$$


In the equation (3.9), the parameter $-\mathrm{dVp} / \mathrm{dIp}$ is called as instantaneous resistance ( $\mathrm{Rp}$ ) and $\mathrm{Vp} / \mathrm{Ip}$ is called as incremental resistance (rp). so the equation no.3.9 can reformulated as

$$
\delta=\mathrm{Rp}-\mathrm{rp}
$$

The difference of these resistance value is defined the sliding surface of MPPT. Whenever $\delta$ value becomes zero which indicates the achievement of maximum power point (MPP). The control law input [16] for MPPT can be defined by the following equation.

$$
X(t)=X e q(t)+X n(t)
$$

Where $\mathrm{Xeq}(\mathrm{t})$ is defined as the system behaviour on sliding surface. $\mathrm{Xn}(\mathrm{t})$ is known as non linear switching inputs. $\mathrm{Xeq}(\mathrm{t})$ is obtained from invariant condition and it is given as

$$
(\delta=0, \mathrm{~d} \delta=0) \text { is biequivalent to }(\mathrm{X}=\mathrm{Xeq})
$$

The derivative of equation (3.10) with respect to time can be written as

$\mathrm{d} \delta=\mathrm{dR} / \mathrm{dt}-\mathrm{dr} / \mathrm{dt}$, then

Multiply both sides by dIp/dt, becomes

$\mathrm{d} \delta=(\mathrm{dR} / \mathrm{dIp}-\mathrm{dr} / \mathrm{Ip}) . \mathrm{dIp} / \mathrm{dt}$

$\mathrm{d} \delta=\omega \mathrm{dIL} / \mathrm{dt}$

where $\quad \mathrm{IL}=\mathrm{K} . \mathrm{Ip}$

Eqn 3.13

where $\mathrm{k}$ is proportional constant.

By substituting equation (3.8) in equation (3.13), the time derivative of sliding surface is obtained as:

$$
d \delta=\omega\left[x(t) \cdot \frac{V_{p}}{L}-(1-x(t)) \cdot \frac{V_{o}}{L}\right]
$$

Considering equation 3.14 and equation 3.12, the equivalent control input is obtained as

$$
\mathrm{Xeq}(\mathrm{t})=\mathrm{Vo} /(\mathrm{Vo}+\mathrm{Vp})
$$

Now $\mathrm{Xn}(\mathrm{t})$ is choosen so that the Lyapunov stability criteria $(\mathrm{d} \delta . \delta<0)$ is met The choosen $\mathrm{Xn}(\mathrm{t})$ as

$$
X_{n}(t)=-\frac{V_{o}}{V_{o}+V_{p}}+\frac{1}{(1+M)}
$$

Where $\mathrm{M}$ is the control signal which is calculated through Lyapunov stability criteria. Then adding the equation (3.15) \&(3.16)

$$
\mathrm{X}(\mathrm{t})=1 /(1+\mathrm{M})
$$

By considering the operating point of system is ' $a$ ' which is shown in figure no. since the gradient is negative, moving the operating point to the right side causes increasing in the current of PV array, which results in decreasing of $\mathrm{Rp}$ and increasing of $\mathrm{rp}$, therefore, $(\partial \mathrm{Rp} / \partial \mathrm{Ip}<0,(\partial \mathrm{rp} / \partial \mathrm{Ip}>0)$. Also, moving the operating point to the left side causes decreasing in the current of PV array, which results in increasing of Rp and decreasing of $\mathrm{rp}$, therefore, $(\partial \mathrm{Rp} / \partial \mathrm{Ip}>0,(\partial \mathrm{rp} / \partial \mathrm{I} \mathrm{p}<0)$. for positive sliding surface $(\delta>0)$;

$$
\frac{V_{p}}{V_{o}}>\left[\frac{V_{p}^{(1-\alpha)} \cdot\left(I_{p}\right)^{\alpha}}{V_{o}}\right]\left|\frac{d V_{p}}{d I_{p}}\right|^{\alpha}
$$

when $(\delta<0)$, 


$$
\frac{V_{p}}{V_{o}}<\left[\frac{V_{p}^{(1-\alpha)} \cdot\left(I_{p}\right)^{\alpha}}{V_{o}}\right]\left|\frac{d V_{p}}{d I_{p}}\right|^{\alpha}
$$

From the equation (3.18) \& (3.19), the control law $\mathrm{M}$ can be choosen as

$$
M=\left[\frac{V_{p}^{(1-\alpha)} \cdot\left(I_{p}\right)^{\alpha}}{V_{o}}\right]\left|\frac{d V_{p}}{d I_{p}}\right|^{\alpha}
$$

Substituting equation (3.20) in equation (3.17), the control input is obtained as

$$
X(t)=\left[1+\left[\frac{V_{p}^{(1-\alpha)} \cdot\left(I_{p}\right)^{\alpha}}{V_{o}}\right]\left|\frac{d V_{p}}{d I_{p}}\right|^{\alpha}\right]^{-1}
$$

The duty cycle must be lie in $0<\mathrm{D}<1$,

$$
\mathrm{D}=\left\{\begin{array}{l}
0, X(t) \leq 0 \\
X(t), 0<X(t)<1 \\
1,1 \leq X(t)
\end{array}\right.
$$

\subsection{Intelligent Technique}

Intelligent MPPT controllers are very robust than conventional nonlinear controllers and it is able to effectively improve the dynamic response and steady state performance of the PV systems. The following section is being reviewed by the main Intelligent algorithms.

\subsubsection{Fuzzy Logic Control}

The Fuzzy logic controller uses the fuzzy logics to make the decisions and to control the output of the controller. The main components in fuzzy logic based MPPT controller are fuzzification, rule-base, inference and defuzzification as shown in figure 8.

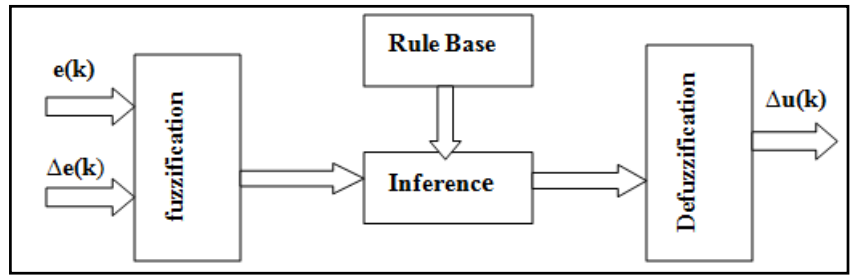

Figure 8. Fuzzy logic block diagram

There are two inputs to the controller - error e $(\mathrm{k})$ and change in error $\Delta \mathrm{e}(\mathrm{k})$. The Fuzzification block converts the crisp inputs to fuzzy inputs. The rules are formed in rule base and are applied in inference block. The defuzzification converts the fuzzy output to the crisp output. The fuzzy inference is carried out by using Mamdani's method [10], and the defuzzification uses the centre of gravity to compute the output of this FLC which is the change in duty cycle.

The inputs to the Fuzzy controller are change in PV array Power ( $\triangle \mathrm{PPV})$ and change in PV array current $(\triangle \mathrm{IPV})$ corresponding to the two sampling time instants. The two inputs are processed by the Fuzzy controller and the output of the Fuzzy controller is the incremental reference current ( $\Delta$ Iref). This output is given to the Dc-Dc power converter. The first input variable $(\Delta \mathrm{Ppv})$ for the fuzzy logic controller is divided into seven Fuzzy sets: PB (Positive Big), PM (Positive Medium), PS (Positive Small), ZZ (Zero), NS 
(Negative Small), NM (Negative Medium) and NB (Negative Big). The second input variable ( $\Delta$ Ipv) for the fuzzy logic controller is divided into 3 Fuzzy sets: $\mathrm{N}$ (Negative), Z (Zero) and P (Positive). The only one output variable ( $\triangle$ Iref) is divided into 7 Fuzzy sets: PB (Positive Big), PM (Positive Medium), PS (Positive Small), ZZ (Zero), NB (Negative Big), NM (Negative Medium) and NS (Negative Small).

The input, output membership functions are shown in figures $9,10,11$ respectively

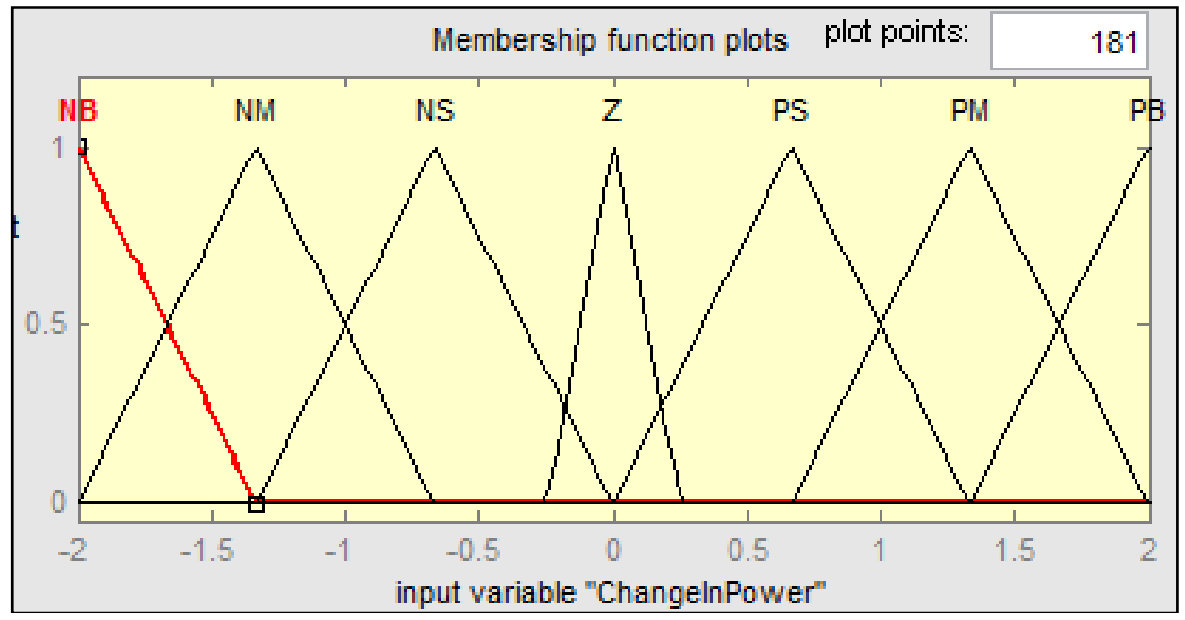

Figure 9. Membership functions of "change in Power" input

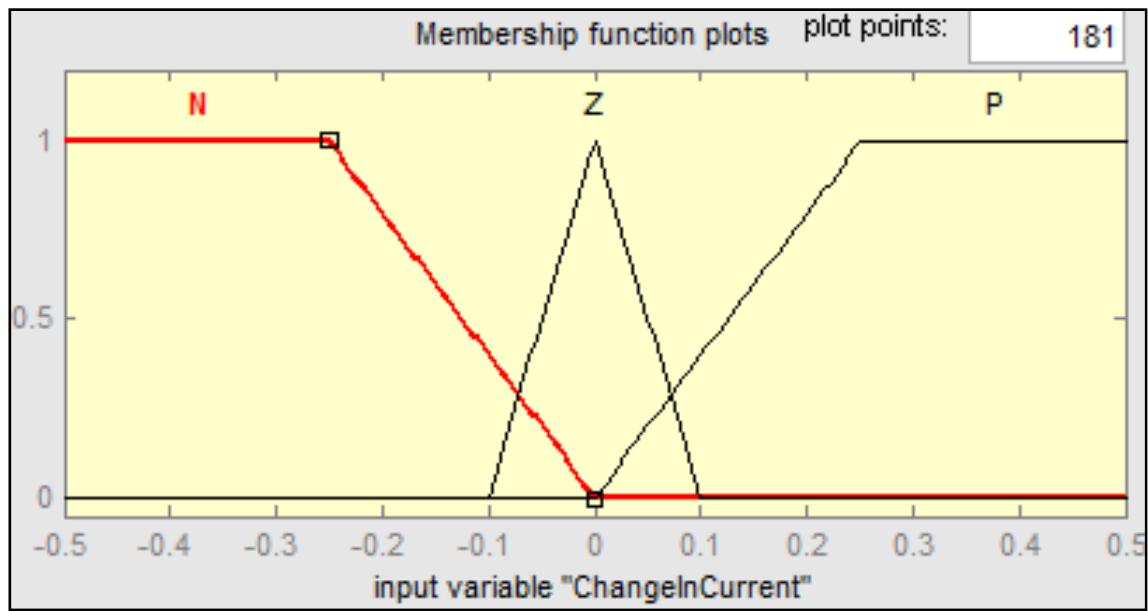

Figure 10. Membership functions of "change in current" input 


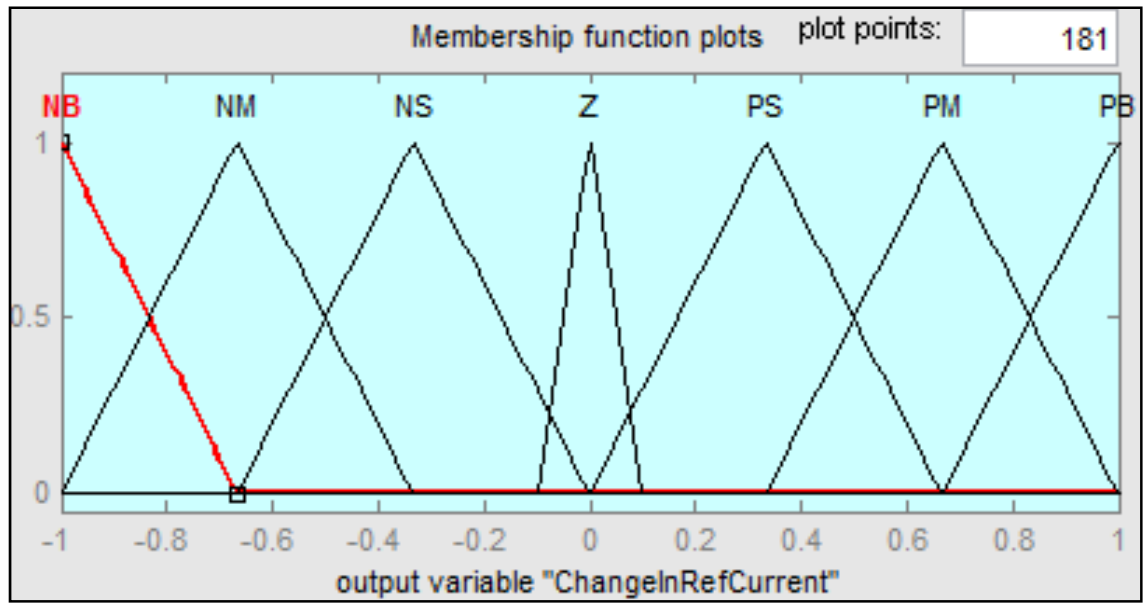

Figure 11. Membership functions of "change in Reference current" output

\subsubsection{Artificial Neural Network (ANN)}

Artificial neural networks (ANN) are best suited for the approximation of non linear systems. It is simply the interconnection of artificial neurons similar to biological brain. Generally it consists of three layers figure12(i.e) input layer, hidden layer and output layer. In each layer, the numbers of nodes are defined by the user and varied based on the requirement.

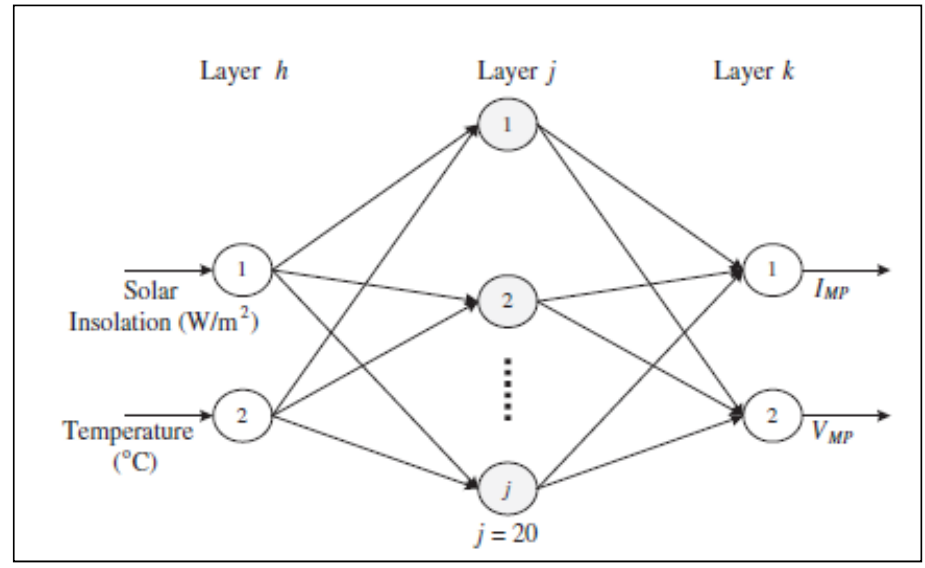

Figure 12. Artificial Neural network structure for MPPT [8]

Feed forward type of neural network is the simplest one and it passes the information only in forward direction to all the layers. The possible configuration of the ANN for PV is shown in figure 12. Normally irradiance $(\mathrm{G})$, Temperature $(\mathrm{T})$ are used as input layer and the output layer may be in the form of voltage, duty cycle depending. The back propagation training method is used. Usually, the training for PV can take few months to ensure the correct response to different atmospheric conditions. During training, the neurons are assigned certain weightage to match the input-output correlation.

\subsubsection{Particle Swarm Optimization (PSO)}

PSO is a stochastic, population based evolutionary algorithm which simulates the behaviors of bird flocking [17] [20]. The algorithm maintains a swarm of individuals as particles. The Figure 13. Shows the movement of particles in the optimization process. The Pbest is the position of a particle, influenced by the best particle in a neighborhood, Gbest is the best solution found by the particle itself, Particle position, xi, is adjusted using 


$$
x_{i}^{k+1}=x_{i}^{k}+v_{i}^{k+1}
$$

where $\mathrm{v}_{\mathrm{i}}$ is the velocity component, represents the step size. Thevelocity is calculated by

$$
v_{i}^{k+1}=w v_{i}^{k}+c_{1} r_{1}\left\{P_{\text {best } i}-x_{i}^{k}\right\}+c_{2} r_{2}\left\{G_{\text {best }}-x_{i}^{k}\right\}
$$

where $\mathrm{w}$ is the inertia weight, $\mathrm{c}_{1}$ and $\mathrm{c}_{2}$ are the acceleration coefficients, $\mathrm{r}_{1}, \mathrm{r}_{2} \in \mathrm{U}(0,1), \mathrm{r}_{1}$ and $\mathrm{r}_{2}$ are random numbers.

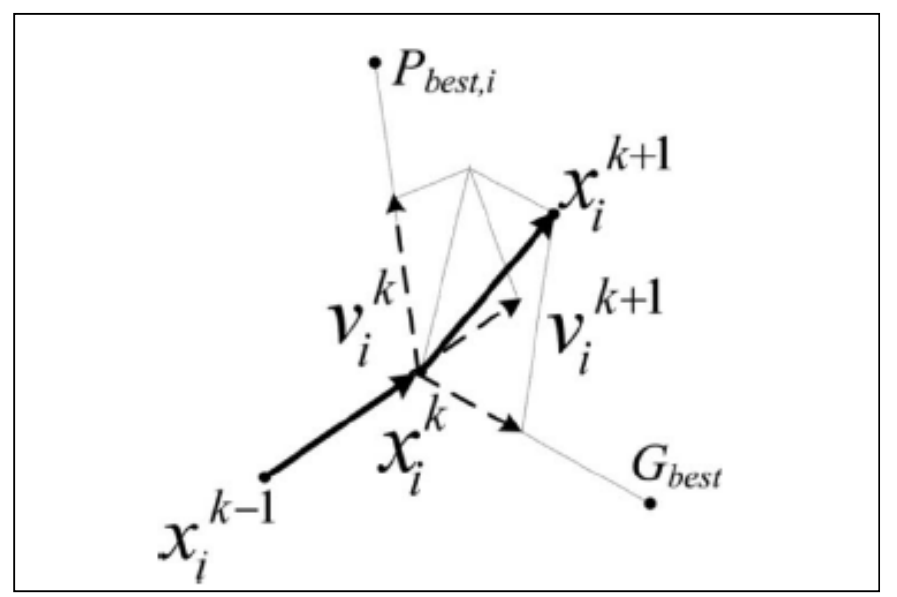

Figure 13. Movement of particles in the optimization process [11]

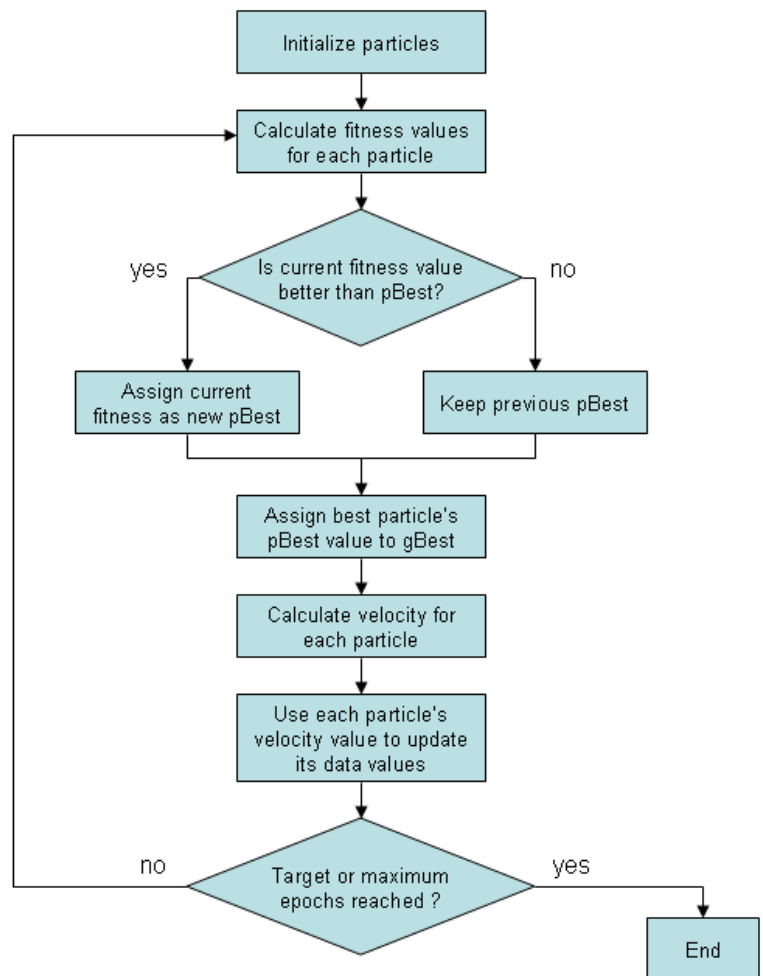

Figure 14. Flow chart of PSO Algorithm [18] 


\section{RESULTS AND ANALYSIS}

In the above section, the classification of main MPPT techniques can be found from the literature reviews. The baseline of all the techniques is to maximize the PV output power regardless of uniform irradiance or partial shading. In this paper, the discussion is made in to the following factors, namely the algorithm complexity, convergence speed, PV array dependency, tracking accuracy, Hardware implementation and its applications. The table 1 depicts the comparison of main MPPT technique algorithms on these factors.

\subsection{Design of Algorithm Complexity}

The efficiency of the MPPT algorithm is fully depends on the design of complexity. It determines how accurate the calculation performed by the algorithm to find the MPP. Otherwise the available solar energy is not completely extracted. Open circuit voltage and short circuit current algorithms are very simple, fast with not much calculations required. But the artificial intelligence based optimization techniques are having highly complex in its algorithm design. Fuzzy Logic, Artificial Neural Network (ANN) and Particle swarm optimization intelligent algorithms are having the advantage of working with imprecise inputs, not requiring accurate mathematical model and handling nonlinearity.

\subsection{Tracking and Convergence Speed}

As the solar irradiation is not uniform for the whole day, it will also vary according to the climatic changes, there is a chance of increase in the formation of partial shading. Due to this partial shading, the PV characteristics will have a multiple peaks formation which leads to immediately affect the tracking efficiency and convergence speed of the PV system. The conventional MPPT algorithms like P\&O and INC cannot track MPP in one step as it requires iterative steps which senses, calculates and perturbs periodically so the tracking is slow and it does not tracking the true MPP. In this context, Fuzzy, ANN and PSO based MPPT algorithms are very sensitive in approach and having the highest capability to track the global peak at very high speed irrespective of the environmental conditions. The designers of PV system, must consider the MPPT algorithm tracking and convergence speed in order to avoid any loss of energy.

\subsection{Implementation}

The choice of selecting the MPPT algorithm for ease of implementation is also an important factor while designing the PV system. However, it highly depends on the user knowledge in handling the MPPT. Because one may prefer to deal with analog circuits and others will more familiar in digital circuits. If the user is interested to work on with analog only, then the open circuit voltage and short circuit current offline technique are the good options. Nevertheless, most of the soft computing intelligence based algorithms like Fuzzy, ANN, PSO, etc are all implemented in digital form which requires the knowledge of programming and software. The implementation of the MPPT algorithm also depends on the complexity design, number of sensors used, costs and other hardware requirements. Some of the MPPT algorithms can be implemented only on the specific topologies.

\subsection{Costs}

In case of rapidly changing environment conditions, the requirement of sensors are more and increases in the complexity design, hardware and costs. It is easier to measure the voltage than the current and current sensors are very expensive. So the current measurement based MPPT techniques requires quite high investment for their hardware implementation and also has fewer losses. The cost of MPPT also depends on the choice of circuits which is either Analog or digital. Comparative to digital, analog circuit costs is low.

\subsection{Applications}

The abovementioned MPPT techniques whatever we discussed so far will be suit for different applications. For example, consider the high end application like space satellites and Orbital stations, the complexity of the algorithm, costs doesn't matter, the only requirement is to track the true MPP within a stipulated time period and no need of periodic tuning. In this type of special applications, $\mathrm{P} \& \mathrm{O}$ and INC are more appropriate. Suppose in the case of large scale PV power plants, the efficiency of the MPPT method constitutes the most important initial consideration and the hybrid MPPT algorithm one is the best option. In the case of designing the solar vehicles would mostly require the convergence speed to the MPP to be very fast. Fuzzy Logic, Artificial neural network, PSO and Slide Mode based MPPT will be preferred for high speed of convergence to the MPP. Open circuit voltage and Short circuit current algorithms will be sufficient 
for the PV systems used for street lightning since it does not require any more tightconstraints and only easy and cheap implementation is required.

Table 1. Comparative characteristics of various MPPT algorithms [4] [5] [8] [12] [19] \& [21]

\begin{tabular}{|c|c|c|c|c|c|c|c|}
\hline $\begin{array}{c}\text { MPPT } \\
\text { Algorithms }\end{array}$ & $\begin{array}{l}\text { Algorithm } \\
\text { Complexity }\end{array}$ & $\begin{array}{c}\text { Convergence } \\
\text { Speed }\end{array}$ & $\begin{array}{c}\text { Analog or } \\
\text { Digital }\end{array}$ & $\begin{array}{c}\text { PV array } \\
\text { dependency }\end{array}$ & $\begin{array}{l}\text { Tracking } \\
\text { Accuracy }\end{array}$ & $\begin{array}{c}\text { Hardware } \\
\text { Implementation }\end{array}$ & Application \\
\hline O.C Voltage & Low & Slow & Both & Yes & Low & Easy & Off Grid \\
\hline S.C Current & Low & Slow & Both & Yes & Low & Easy & Off Grid \\
\hline P\&O & Low & Slow & Both & No & Medium & Easy & Both \\
\hline INC & Medium & Fast & Digital & No & High & Moderate & Both \\
\hline Fuzzy & Medium & Fast & Digital & Yes & Very High & Easy & Both \\
\hline ANN & High & Fast & Digital & Yes & Very High & Difficult & Grid \\
\hline PSO & High & Fast & Digital & No & Very High & Moderate & Both \\
\hline Slide Mode & Medium & Fast & Digital & No & Medium & Difficult & Both \\
\hline
\end{tabular}

\section{CONCLUSION}

The paper reviews the various MPPT algorithm techniques that have been used for Photovoltaic system at changes in irradiance and temperature. It highlights the technical aspects, merits, demerits and analyzes the performance of each method. The selection of a particular MPPT technique is application specific and analyzed the current technique in this article. Based on this review, it can be concluded that the offline and online techniques are working very well only when the solar irradiation is uniform. But it failed to approach the true MPP when it comes under rapidly changing atmospheric and partial shaded conditions. This drawback was overcome by the new MPPT techniques based on artificial intelligence which exhibits good performance in tracking the MPP. Still more researches are moving to find a simple, low cost, easily implementable and highly efficient algorithms. Hence in the near future, the combination of either direct/indirect technique algorithms with intelligence technique hybrid algorithms will hope to perform a perfect tracker for MPP under all irradiance conditions.

\section{REFERENCES}

[1] Hiren Patel and Vivek Agarwal, "Maximum Power Point Tracking Scheme for PV Systems Operating Under Partially Shaded Conditions", IEEE Transactions on Industrial Electronics, Vol. 55, No. 4, April 2008.

[2] Hussein $\mathrm{KH}$, et al. "Maximum photovoltaic power tracking: an algorithm for rapidly changing atmospheric conditions", IEEE Proceedings on Generation, Transmission and Distribution 1995; 142:59-64.

[3] Moacyr Aureliano Gomes de Brito, Luigi Galotto, Jr., Leonardo Poltronieri Sampaio, Guilherme de Azevedo e Melo, and Carlos Alberto Canesin, "Evaluation of the Main MPPT Techniques for Photovoltaic Applications", IEEE Transactions On Industrial Electronics, Vol. 60, No. 3, March 2013.

[4] Trishan Esramand Patrick L. Chapman, "Comparison of Photovoltaic Array Maximum Power Point Tracking Techniques", IEEE Transactions on Energy Conversion, Vol. 22, No. 2, June 2007.

[5] Pallavee Bhatnagar, R.K. Nema "Maximum powerpoint tracking control techniques: State-of-the-art in photovoltaic applications", Science Direct, Renewable and Sustainable Energy Reviews 23 (2013) 224-241

[6] "Photovoltaic Modeling", Power Analytics Corporation16870 West Bernardo Drive, Suite 330 San Diego, CA 92127, U.S.A.

[7] N. Pandiarajan and Ranganath Muthu, "Mathematical Modeling of Photovoltaic Module with Simulink", International Conference on Electrical Energy Systems (ICEES 2011), 3-5 Jan 2011.

[8] Zainal Salam, Jubaer Ahmed, Benny S. Merugu, "The application of soft computing methods for MPPT of PV system: A technological and status review”, Science Direct, Applied Energy, 107 (2013) 135-148.

[9] Saravana Selvan. D, Harikrishnan. V, Umayal. V, Indumathy, "Performance Analysis of slide Mode Control based MPPT Controller for Photovoltaic Applications", in the "International Conference on Advances in Engineering and Technology (ICAET'2014)", Conference proceedings ISBN 978-93-82242-85-7, Singapore on March 29-30, 2014.

[10] Saravana Selvan. D, Mohammed feros khan. J, Umayal. V, Indumathy. M, "Simulation of Fuzzy Logic control based MPPT Technique for Photovoltaic System", in the "2nd International Conference on Innovations in Engineering and Technology (ICCET '2014)”, Conference proceedings ISBN 978-81-929653-6-9, Penang on Sept 19-20, 2014.

[11] Kashif Ishaque and Zainal Salam, "A Deterministic Particle Swarm Optimization Maximum Power Point Tracker for Photovoltaic System Under Partial Shading Condition”, IEEE transactions on Industrial Electronics, vol. 60, no. 8, august 2013.

[12] Ali Reza Reisi, Mohammad Hassan Moradi, Shahriar Jamasb, "Classification and comparison of maximum power point tracking techniques for photovoltaic system: A review", Science Direct, Renewable and Sustainable Energy Reviews 19 (2013) 433-443.

[13] M.D Goudar, B.P Patil, V. Kumar, “ A Review of Improved maximum Peak Power tracking Algorithms For Photovoltaic Systems", International Journal of electrical Engineering and technology (IJEET), ISSN 0976-6545. 
[14] Adel Mellit, Soteris, Kalogirou, "Artificial intelligence techniques for photovoltaic applications: A review", Science Direct, Progress in Energy and Combustion Science, 34 (2008) 574-632.

[15] Lijun Qin, Xiao Lu, "Matlab/Simulink-Based Research on Maximum Power Point Tracking of Photovoltaic Generation", Science Direct, Physics Procedia, 24 (2012) 10 - 18.

[16] II-Song K., "Sliding mode controller for the single-phase grid connected photovoltaic system", Applied Energy, Vol. 83, pp. 1101-1115, October 2006.

[17] R. Eberhart and J. Kennedy, "A new optimizer using particle swarm theory”, in Proc. 6th Int. Symp. MHS, 1995, pp. 39-43.

[18] http://www.mnemstudio.org/particle-swarm-introduction.htm

[19] http://link.springer.com/chapter/10.1007/978-3-642-37105-9 6

[20] http://waset.org/Publication/multiple-peaks-tracking-algorithm-using-particle-swarm-optimization-incorporatedwith-artificial-neural-network $/ 280$

[21] http://www.cscanada.net/index.php/est/article/view/j.est.1923847920130602.2671

[22] Hohm D, Ropp M., "Comparative study of maximum power point tracking algorithms Progress in photovoltaics", Research and Applications, 2003; 11: 47-62.

[23] Her-TerngYau, Qin-Cheng Liang, Chin-Tsung Hsieh, "Maximum power point tracking and optimal Li-ion battery charging control for photovoltaic charging system", Science direct, Computers and Mathematics with Applications, 64 (2012) 822-832.

[24] Khaehintung, Noppadol, Theerayod Wiangtong, and Phaophak Sirisuk, "FPGA implementation of MPPT using variable step-size P\&O algorithm for PV applications", Communications and Information Technologies ISCIT'06", International Symposium on IEEE, 2006.

[25] Safari, Azadeh, and Saad Mekhilef, "Simulation and hardware implementation of incremental conductance MPPT with direct control method using Cuk converter", Industrial Electronics, IEEE Transactions on, 58.4 (2011): 11541161.

[26] Chekired, F., et al., "Intelligent maximum power point trackers for photovoltaic applications using FPGA chip: Acomparative study", Solar Energy, 101 (2014): 83-99. 